\title{
Malaria vaccine protects offspring
}

\author{
from F.E.G. Cox
}

THERE can be no doubt that an antimalarial vaccine is one of the major requirements of tropical medicine and a number of laboratories are attempting to develop one. There is equally no doubt that vaccination against malaria is possible and has been achieved using a wide range of experimental model systems, but the protection produced varies in efficacy and all the methods so far described have serious drawbacks.

In the life cycle of the malaria parasite in the human host, the infective stages injected by the mosquito are the sporozoites, which circulate in the body for about half an hour before entering the liver where a massive phase of multiplication occurs. This results in the production of thousands of merozoites which invade red blood cells where another multiplication phase, erythrocytic schizogony, takes place. The products of this phase are further merozoites which invade fresh red blood cells in which erythrocytic schizogony again occurs and this process is repeated until the host dies or recovers. Eventually sexual stages are produced and these are taken up by and infect the mosquito vector ${ }^{1}$.

The various stages in the life cycle differ antigenically from one another; thus as the immunity produced is stage specific, a blockbuster approach to immunization is not feasible. At present the main targets for vaccines are the sporozoites, the merozoites, the erythrocytic stages and the gametocytes ${ }^{2}$. The use of irradiated sporozoites as a vaccine has been largely confined to rodent models but with considerable success. Adult mice can be protected by the intravenous injection of irradiated sporozoites ${ }^{3}$. The protective antigen is a surface antigen that can be demonstrated with the use of monoclonal antibodies ${ }^{4}$ and monoclonal antibodies against this antigen protect mice against challenge with the homologous species ${ }^{5}$. On page 331 of this issue of Nature Orjih and colleagues report that these findings have been extended to young mice and the offspring of immunized mothers.

Briefly, adult mice given one to four immunizing doses of irradiated sporozoites intramuscularly were poorly protected against challenge with live sporozoites several weeks later. Young mice 2-14 days old, on the other hand, were well protected. The fact that adult mice can be protected by the intravenous route but not the intramuscular one is interesting but what these experiments do show is that young animals can be immunized in an acceptable way. Of even greater impor-

F.E.G. Cox is Professor of Zoology at King's College London. tance is the fact that when adult female mice were immunized intravenously with irradiated sporozoites, over half the offspring were protected against challenge and similar levels of protection were found in mice born to normal mothers but fostered onto immunized ones.

These results are particularly important because, in many parts of the world, malaria is essentially a disease of children. In endemic areas, babies acquire immunity passively from their mothers and are then protected during the critical first few months of life. Control schemes, however, upset this balance and it is in such situations that a vaccine that protects young children is likely to be of most use. Another important aspect of these results is that similar findings may apply to other vaccines such as the merozoite one being developed at Guy's Hospital in London ${ }^{2}$, and recently described improvements in the in vitro cultivation of human malaria parasites $^{6.7}$ and adjuvants ${ }^{8}$ have moved such a vaccine a further step forwards. The merozoite vaccine is known to protect monkeys but for obvious reasons the possibility of transfer of immunity to their offspring has not yet been investigated.

Those who have advanced the cause of a malaria vaccine have never suggested that a totally effective vaccine would ever be possible but have argued that a vaccine could produce better protection than a natural infection and could ameliorate the course of the disease. These experiments with Plasmodium berghei appear to vindicate this cautious approach. However, before too much euphoria sets in it must be remembered that the passage of immunity from mothers to offspring differs markedly from species to species and what happens in a mouse or monkey need not necessarily happen in man.

Cox, F.E.G. Biologist 28, 9 (1981).

Cohen, S. Proc. R. Soc. B203, 323 (1979).

Orjih, A.U. \& Nussenzweig, R.S. Am. J. trop. Med. Hyg. 29, $343(1980)$.

Yoshida, N et al Science 207, 71 (1980)

Potocnjak, P. et al. J. exp. Med. 51, 1504 (1980)

Butcher, (i. A. Ann. trop. Med. Parasit. 75, 111 (1981)

Scott-Finnigan, T.J., Wilson, R.J.M. \& Williamson, J. Trans. R. Soc. trop. Med. Hyg. 75, 292 (1981).

8. Siddiqui, W.A. et al. Nature 289, 64 (1981).

\section{Continental swells}

\section{from Peter J. Smith}

As newly formed lithosphere spreads away from oceanic ridges it cools and contracts, leading to a subsidence of the ocean floor that increases generally with age. Indeed, as Sclater and colleagues have shown $(J$. geophys. Res. 76; 7888, 1971), there is a simple relationship between lithospheric age and water depth, which may be regarded as the first-order description of ocean floor elevation. However, as with most geological phenomena, there are also second-order and even third-order effects - local deviations from the general rule. Chief among these are the broad topographical swells, typically $1,000 \mathrm{~km}$ or so across and about $1 \mathrm{~km}$ high, associated with zones of extensive intraplate volcanism such as the Canaries, the Cape Verde Islands and Hawaii, as well as the topographical highs representing aseismic ridges such as the Rio Grande Rise and volcanically long extinct regions such as Bermuda.

Cochran and Talwani (Geophys. J. 50; 495,1977 ) have recently shown that both

Peter J. Smith is in the Department of Earth Sciences, The Open University, UK. the volcanically active topographical features (known in certain quarters as hot spots) and their inactive counterparts are associated with positive gravity anomalies of intermediate wavelength - an observation that poses certain problems of interpretation, especially if volcanic activity alone is to be regarded as the lithospheric manifestation of mantle plumes. Similar gravity effects suggest common underlying causes; and yet it is not entirely clear how topographical anomalies associated with volcanic activity, whether plumerelated or not, could be supported by the same mechanism as, say, aseismic ridges. Detrick and Crough (J, geophys. Res. 83; 1236,1978 ) may or may not be right, therefore, in attributing the 'active' topographical swells to reheating of the lithosphere.

Whatever the mechanism may be, what produces little more than a topographical swell in oceanic lithosphere may produce rather more complex results in continental lithosphere. This supposition has led Crough (Geology 9; 1, 1981) to examine the consequences of a possible topographical swell moving across parts of the United 